\title{
Hospital variation in admissions to neonatal intensive care units by diagnosis severity and category
}

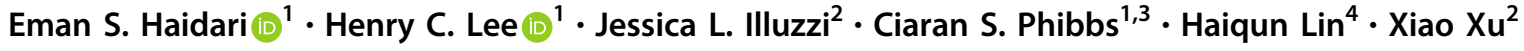

Received: 21 February 2020 / Revised: 17 July 2020 / Accepted: 3 August 2020 / Published online: 14 August 2020

(C) The Author(s), under exclusive licence to Springer Nature America, Inc. 2020

\begin{abstract}
Objective To examine interhospital variation in admissions to neonatal intensive care units (NICU) and reasons for the variation.

Study design 2010-2012 linked birth certificate and hospital discharge data from 35 hospitals in California on live births at $35-42$ weeks gestation and $\geq 1500 \mathrm{~g}$ birth weight were used. Hospital variation in NICU admission rates was assessed by coefficient of variation. Patient/hospital characteristics associated with NICU admissions were identified by multivariable regression.

Results Among 276,489 newborns, 6.3\% were admitted to NICU with 34.5\% of them having mild diagnoses. There was high interhospital variation in overall risk-adjusted rate of NICU admission (coefficient of variation $=26.2$ ) and NICU admission rates for mild diagnoses (coefficient of variation: 46.4-74.0), but lower variation for moderate/severe diagnoses (coefficient of variation: 8.8-14.1). Births at hospitals with more NICU beds had a higher likelihood of NICU admission. Conclusion Interhospital variation in NICU admissions is mostly driven by admissions for mild diagnoses, suggesting potential overuse.
\end{abstract}

\section{Introduction}

Trends toward increasing neonatal intensive care unit (NICU) admissions of neonates with higher gestational age, greater birth weight, and lower illness acuity over time have raised concern for potential misuse of NICUs [1]. In 2015, nearly $80 \%$ of all NICU admissions in California were for infants $\geq 34$ weeks gestational age,

Supplementary information The online version of this article (https:// doi.org/10.1038/s41372-020-00775-z) contains supplementary material, which is available to authorized users.

Eman S. Haidari

ehaidari@stanford.edu

1 Department of Pediatrics, Stanford University School of Medicine, Stanford, CA, USA

2 Department of Obstetrics, Gynecology and Reproductive Sciences, Yale School of Medicine, New Haven, CT, USA

3 Health Economics Resource Center, Veterans Affairs Palo Alto Health Care System, Palo Alto, CA, USA

4 Division of Nursing Science, Rutgers School of Nursing, Rutgers University, Newark, NJ, USA and only $12 \%$ were classified as having conditions indicative of high illness acuity [2]. A recent populationbased study from the Vermont Oxford Network including nearly half a million infants showed similar results [3]. In addition, multiple studies have demonstrated large variation in NICU admission rates across hospitals [3-5], with a 34-fold difference in NICU inborn admission rates found in California [2]. Such large variation also raises concern for potential overuse of NICUs. However, little is known about why this variation exists, as research on large population data exploring reasons for NICU admissions is sparse.

NICU admission not only comes with a hefty and increasing economic burden [6], there are also iatrogenic costs such as medication errors [7] and increased infection risk [8] to be considered, as well as barriers to maternal-infant bonding and breastfeeding that result in lower long-term breastfeeding rates [9-11] with associated implications for pediatric health. Given the risks associated with NICU admission, strategies to optimize utilization of NICUs are needed. A better understanding of variation in NICU admission rates across hospitals and reasons for the variation may help to identify areas of excess utilization and potential remedies. 
In that vein, we used linked birth certificate and hospital discharge data to identify babies admitted to the NICU in a sample of Californian hospitals and examined their diagnosis codes to explore reasons for NICU admission. Through this method, we were able to evaluate not only variation in overall admission rates across hospitals, but also across defined diagnosis severity categories in an attempt to identify potential diagnoses where overutilization may occur.

\section{Materials and methods}

\section{Data source and study sample}

This study used data from the 2010 to 2012 linked birth file from California that contained information from birth certificates as well as hospital discharge records [12]. The algorithm used to link the birth certificate and hospital discharge data has previously been shown to achieve a linkage rate of $98.59 \%$ [13]. We limited our sample to live singleton births at 35-42 weeks gestation with a birth weight of at least $1500 \mathrm{~g}$ that had birth certificates successfully linked to hospital discharge records. This birth weight cutoff was chosen since infants below $1500 \mathrm{~g}$ are routinely admitted to NICUs, while infants with birth weight above $1500 \mathrm{~g}$ comprise a large portion of NICU admissions for which no routine admission guidelines are available and thus, there is likely more variability in hospital practice. Births with a disposition status of "Left against medical advice or discontinued care" were excluded. A previous study evaluated accuracy of NICU admission rates documented on birth certificates in California and identified 35 hospitals as providing optimal quality data [14]. In order to ensure accuracy of our measurement of NICU admissions, we further limited our sample to eligible inborn births at these 35 hospitals. We used data from 2010 to 2012, which were the latest years that the linked birth file was available. A total of 276,489 births met the sample eligibility criteria. This study was approved by Yale University Human Investigation Committee and the California Committee for the Protection of Human Subjects.

\section{Measures}

For each infant, we measured whether he/she was admitted to the NICU based on an indicator on the birth certificate reporting whether "NICU admission" occurred. According to birth certificate reporting guidelines from the Centers for Disease Control and Prevention, this item reflects "admission into a facility or unit staffed and equipped to provide continuous mechanical ventilator support for a newborn," and does not include "well-baby nurseries or special care nurseries (i.e., Level II nursery)" [15]. The 35 hospitals included in this study all had a level III or IV NICU, and were similar in characteristics when compared with other hospitals with a level III-IV NICU [14]. Recognizing that birth certificates tended to underreport events including NICU admissions [14], we supplemented this data item with the following information sources: (1) "Assisted ventilation for more than $6 \mathrm{~h}$ " (birth certificate), (2) "Newborn given surfactant replacement therapy" (birth certificate), (3) "Antibiotics received by the newborn for suspected neonatal sepsis" (birth certificate), (4) "Seizures or serious neurologic dysfunction" (birth certificate), (5) International Classification of Diseases Ninth Revision (ICD-9) procedure codes indicative of procedures that were typically administered in NICUs (such as continuous invasive mechanical ventilation, spinal tap, and umbilical vein catheterization), (6) hospital disposition status indicative of death or transfer out, and (7) diagnosis related group code indicative of death, transfer out or extended use of mechanical ventilation. Infants that had the birth certificate indicator for "NICU admission" or any of the aforementioned supplemental indicators were considered as having been admitted to the NICU.

To assess reasons for NICU admissions, we classified each infant as having severe, moderate, or mild conditions. We defined severe and moderate conditions following the categories of severe and moderate unexpected newborn complications developed by the California Maternal Quality Care Collaborative, which was a perinatal care quality measure endorsed by the National Quality Forum and used by The Joint Commission for tracking hospital performance [16-18]. Severe conditions included severe birth trauma, hypoxia/asphyxia, shock/resuscitation, severe infection/ sepsis, severe respiratory complications, and severe neurological complications, as well as in-hospital death and transfer out. Moderate conditions included less serious but significant birth trauma, infection, respiratory complications, and neurological complications, as well as length of stay longer than 5 days (except for those with jaundice or social problems). These conditions were defined based on ICD-9 diagnosis and procedure codes, length of stay, and disposition status. Only infants that did not experience any of the severe conditions were coded for moderate conditions. Among infants that did not have severe or moderate conditions, we further identified the occurrence of five mild diagnoses determined to likely have borderline admission criteria: mild respiratory conditions, suspected infection, hypoglycemia, drug exposure/neonatal abstinence syndrome (NAS), and hyperbilirubinemia (see Appendix A for detailed definition of these mild conditions). These mild conditions were not mutually exclusive. Infants who did not meet these definitions for severe, moderate, or the five selected mild conditions were classified as "other." 
Using combined information from birth certificate and hospital discharge record, we measured each infant's race/ ethnicity, primary payer, gestational age, parity, birth weight, Apgar score at $5 \mathrm{~min}$, maternal age, maternal body mass index category, length of stay, and hospital charges. Characteristics of the delivery hospital included teaching status, type of ownership, affiliation with a multihospital system, level of NICU, and number of NICU beds. Data on these hospital characteristics were obtained from the 2011 American Hospital Association annual survey database [19] and the California Perinatal Quality Care Collaborative [20]. For NICU beds, we also calculated a standardized measure reflecting the number of NICU beds per 1000 live inborn births based on the average annual birth volume of each hospital in our study period.

Patient and hospital characteristics were summarized using descriptive statistics and were compared by NICU admission status using chi-square test for categorical variables and Student's $t$ test for continuous variables (or Wilcoxon rank sum test if the continuous variable was not normally distributed). Among infants admitted to the NICU, we calculated the proportion of them having either a severe condition, moderate condition, or any of the mild conditions, in order to inform the most common reasons for NICU admissions.

To examine differences in NICU admission rates across hospitals, we first assessed hospital variation in overall rate of NICU admissions. For this, we used a hierarchical generalized linear model to estimate the likelihood of NICU admission, while adjusting for patient clinical risk factors (including maternal age, maternal body mass index category, gestational age, parity, birth weight category, Apgar score, congenital malformations, and severity of diagnostic groups) and accounting for a hospital-specific random effect. This allowed us to calculate a risk-adjusted rate of overall NICU admission for each hospital by dividing its sum of predicted NICU admissions (conditional on its patient clinical risk factors and hospital random effect) by its sum of expected NICU admissions (conditional on its patient clinical risk factors without hospital random effect), and multiplying by the observed rate of overall NICU admissions in the entire sample. Next, we assessed hospital variation in observed NICU admission rates stratified by diagnosis category (limited to hospitals that had at least 25 infants with a given diagnosis category in order to ensure adequate sample size for assessing their practice) [21]. Variation in NICU admission rate across hospitals was assessed using coefficient of variation. Following prior research [5], we classified coefficients of variation less than 10 as indicating low variation, coefficients of variation between 10 and less than 20 as medium variation, and coefficients of variation greater than or equal to 20 as high variation. Correlation of a hospital's NICU admission rates between the different diagnosis categories were assessed using Spearman correlation coefficient.

We also performed a multivariable regression analysis to inform nonclinical factors that may influence the likelihood of NICU admissions. We focused the analysis on infants with mild conditions where NICU admissions could be more discretionary or ambiguous. We used NICU admission (yes/no) as the dependent variable and patient sociodemographic and hospital characteristics as key explanatory variables, while adjusting for patient clinical risk factors. A hierarchical generalized linear model was used with a logit link function and binomial distribution, as well as a hospital random effect to account for clustering of births by hospital. $P$ values $<0.05$ were considered statistically significant. All analysis was conducted using SAS 9.4 (SAS Institute Inc., Cary, NC).

\section{Results}

Among the 276,489 newborns meeting our sample eligibility criteria, $17,529(6.3 \%)$ were admitted to the NICU. Compared to newborns not admitted to NICUs, those who were admitted were significantly more likely to have lower birth weight, earlier gestational age, lower maternal parity, congenital malformation, or 5 min Apgar score $<7$ (Table 1). They also had a longer length of stay and higher hospital charge. In addition, a higher proportion of newborns who were admitted to NICUs were delivered at teaching institutions, hospitals with a multihospital affiliation, level IV hospitals, and hospitals with more NICU beds.

The rate of NICU admission varied amongst the 35 hospitals examined even after adjusting for differences in patient clinical risk factors (Fig. 1). Median risk-adjusted rate of overall NICU admission was $6.3 \%$ (interquartile range: $5.1-7.6 \%$ ). The coefficient of variation in riskadjusted NICU admission rate was 26.2, suggesting high variation.

Many newborns admitted to NICUs had either a severe $(30.9 \%)$ or moderate $(23.0 \%)$ condition; however, a substantial proportion of the admitted newborns (34.5\%) had mild conditions (Fig. 2a). In particular, $16.9 \%$ of the admitted newborns had a mild respiratory condition, $17.5 \%$ had suspected infection, and $13.9 \%$ had hyperbilirubinemia (Fig. 2b). Twelve percent of the newborns had two mild conditions, and $2.9 \%$ had at least three mild conditions (Fig. 2b). A hospital's risk-adjusted rate of overall NICU admission was positively correlated with the proportion of its NICU admissions being for mild conditions (Spearman correlation coefficient $=0.36$, $p=0.03)$.

Among newborns with severe and moderate conditions, $87.9 \%$ and $72.4 \%$, respectively, were documented as 
Table 1 Characteristics of newborns.

\begin{tabular}{|c|c|c|c|c|}
\hline \multirow[t]{3}{*}{ Characteristics } & \multirow{3}{*}{$\begin{array}{l}\text { Overall } \\
N(\%)\end{array}$} & \multicolumn{3}{|c|}{ NICU admission status } \\
\hline & & Yes & No & $P$ value \\
\hline & & $N(\%)$ & $N(\%)$ & \\
\hline Sample size & 276,489 & 17,529 & 258,960 & \\
\hline $\begin{array}{l}\text { Maternal age (years), mean } \pm S D \text {, } \\
\text { median (IQR) }\end{array}$ & $\begin{array}{l}29.1 \pm 6.2 \\
29(25-34)\end{array}$ & $\begin{array}{l}29.2 \pm 6.5 \\
29(24-34)\end{array}$ & $\begin{array}{l}29.1 \pm 6.2 \\
29(25-34)\end{array}$ & 0.32 \\
\hline Race/ethnicity & & & & $<0.001$ \\
\hline Non-Hispanic white & $99,803(36.1)$ & $6514(37.2)$ & $93,289(36.0)$ & \\
\hline Non-Hispanic black & $14,645(5.3)$ & $1073(6.1)$ & $13,572(5.2)$ & \\
\hline Hispanic & $112,996(40.9)$ & $6805(38.8)$ & $106,191(41.0)$ & \\
\hline Other $^{\mathrm{a}}$ & $49,045(17.7)$ & $3137(17.9)$ & 45,908 (17.7) & \\
\hline Primary payer & & & & $<0.001$ \\
\hline Private insurance & $144,539(52.3)$ & $8672(49.5)$ & $135,867(52.5)$ & \\
\hline Medicaid & $122,216(44.2)$ & $7569(43.2)$ & $114,647(44.3)$ & \\
\hline Self-pay & $3893(1.4)$ & $213(1.2)$ & $3680(1.4)$ & \\
\hline Other & $5841(2.1)$ & $1075(6.1)$ & $4766(1.8)$ & \\
\hline Gestational age (weeks) & & & & $<0.001$ \\
\hline $35-36$ & $13,075(4.7)$ & $3761(21.5)$ & $9314(3.6)$ & \\
\hline $37-40$ & $242,200(87.6)$ & $12,333(70.4)$ & $229,867(88.8)$ & \\
\hline $41-42$ & $21,214(7.7)$ & $1435(8.2)$ & $19,779(7.6)$ & \\
\hline Maternal body mass index & & & & $<0.001$ \\
\hline Underweight & $10,541(3.8)$ & $579(3.3)$ & $9962(3.8)$ & \\
\hline Normal & $136,076(49.2)$ & $7836(44.7)$ & $128,240(49.5)$ & \\
\hline Overweight & $66,539(24.1)$ & $4227(24.1)$ & $62,312(24.1)$ & \\
\hline Obese & $43,476(15.7)$ & $3058(17.4)$ & $40,418(15.6)$ & \\
\hline Morbidly obese & $7281(2.6)$ & $674(3.8)$ & $6607(2.6)$ & \\
\hline Unknown & $12,576(4.5)$ & $1155(6.6)$ & $11,421(4.4)$ & \\
\hline Birth weight (grams) & & & & $<0.001$ \\
\hline $1500-2499$ & $8790(3.2)$ & $2668(15.2)$ & $6122(2.4)$ & \\
\hline 2500-3999 & $244,529(88.4)$ & $13,175(75.2)$ & $231,354(89.3)$ & \\
\hline$\geq 4000$ & $23,170(8.4)$ & $1686(9.6)$ & $21,484(8.3)$ & \\
\hline Parity & & & & $<0.001$ \\
\hline 0 & $112,997(40.9)$ & $9076(51.8)$ & $103,921(40.1)$ & \\
\hline 1 & $90,240(32.6)$ & $4309(24.6)$ & $85,931(33.2)$ & \\
\hline$\geq 2^{\mathrm{a}}$ & $73,252(26.5)$ & $4144(23.6)$ & $69,108(26.7)$ & \\
\hline Apgar score at $5 \mathrm{~min}$ & & & & $<0.001$ \\
\hline $0-6$ & $1576(0.6)$ & $1111(6.3)$ & $465(0.2)$ & \\
\hline $7-10$ & $274,158(99.2)$ & $16,252(92.7)$ & 257,906 (99.6) & \\
\hline Unknown & $755(0.3)$ & $166(0.9)$ & $589(0.2)$ & \\
\hline Congenital malformations & & & & $<0.001$ \\
\hline Yes & $8737(3.2)$ & $2914(16.6)$ & $5823(2.2)$ & \\
\hline No & $267,752(96.8)$ & $14,615(83.4)$ & $253,137(97.8)$ & \\
\hline $\begin{array}{l}\text { Length of stay (days), mean } \pm \\
\text { SD, median (IQR) }\end{array}$ & $2.5 \pm 2.8,2(2,3)$ & $6.4 \pm 9.4,4(2,7)$ & $2.2 \pm 1.1,2(2,3)$ & $<0.001$ \\
\hline $\begin{array}{l}\text { Hospital charge } \mathrm{e}^{\mathrm{b}}(\$), \text { mean } \pm \mathrm{SD} \text {, } \\
\text { median }(\mathrm{IQR})\end{array}$ & $\begin{array}{l}9468 \pm 64,190 \\
4037(2793,6049)\end{array}$ & $\begin{array}{l}77,669 \pm 246,708 \\
33,081(10,247 \\
67,493)\end{array}$ & $\begin{array}{l}4969 \pm 7090 \\
3904(2734,5685)\end{array}$ & $<0.001$ \\
\hline
\end{tabular}


Table 1 (continued)

\begin{tabular}{llll}
\hline Characteristics & Overall & NICU admission status & No \\
\cline { 2 - 3 } & & Yes & $N(\%)$ \\
\hline Teaching status & & $N(\%)$ & $137,727(53.2)$ \\
$\quad$ Teaching & $149,077(53.9)$ & & $121,233(46.8)$ \\
$\quad$ Non teaching & $127,412(46.1)$ & $11,350(64.7)$ & $<0.001$ \\
Type of ownership & & $6179(35.3)$ & $33,555(13.0)$ \\
$\quad$ Government (non federal) & $35,618(12.9)$ & $2063(11.8)$ & $209,153(80.8)$ \\
$\quad$ Private non profit & $223,428(80.8)$ & $14,275(81.4)$ & $16,252(6.3)$ \\
$\quad$ Private for-profit & $17,443(6.3)$ & $1191(6.8)$ & $189,865(73.3)$ \\
Multihospital system affiliation & & $14,235(81.2)$ & $69,095(26.7)$ \\
$\quad$ Yes & $204,100(73.8)$ & $3294(18.8)$ & $<0.001$ \\
No & $72,389(26.2)$ & & $230,403(89.0)$ \\
Level of NICU & & $14,135(80.6)$ & $28,557(11.0)$ \\
$\quad$ III & $244,538(88.4)$ & $3394(19.4)$ & $28.3 \pm 18.7$,
\end{tabular}

IQR interquartile range, NICU neonatal intensive care unit, $S D$ standard deviation.

aNewborns with unknown race/ethnicity were included in the "Other" category and newborns with unknown parity were included in the " $\geq 2$ " category to protect confidentiality and avoid reporting small cells (i.e., cells containing $\leq 15$ patients).

${ }^{\mathrm{b}} N=28,817$ births had missing data on charges.

Fig. 1 Variation in neonatal intensive care admission rate across hospitals $(N=35)$.

Distribution of risk-adjusted NICU admission rate across hospitals for live singleton births with 35-42 weeks gestation and birth weight $\geq 1500$ grams. NICU neonatal intensive care unit.

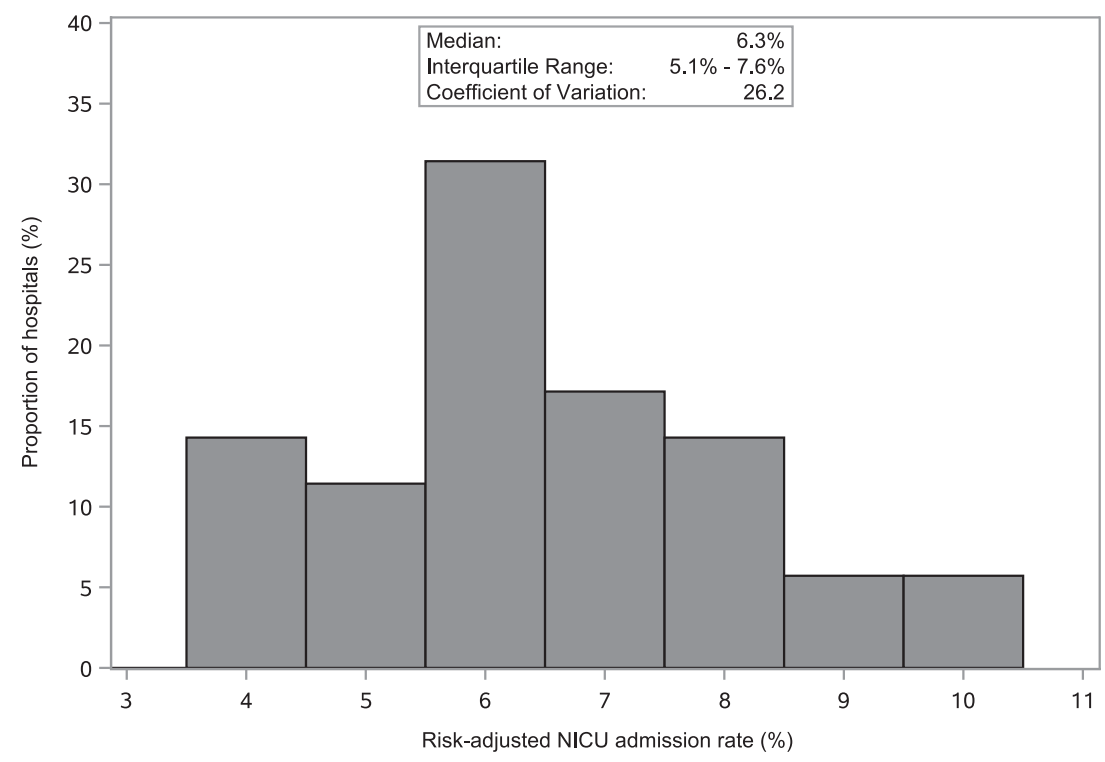

admitted to the NICU with low (coefficients of variation: 8.8) and medium (coefficients of variation: 14.1) variation across hospitals, respectively (Table 2). Among newborns with mild conditions, the rates of NICU admission were substantially lower, ranging from $4.6 \%$ for hyperbilirubinemia to $39.6 \%$ for mild respiratory condition.
However, NICU admission rates for mild conditions had high variation across hospitals. The largest interhospital variations were observed in suspected infection (coefficient of variation: 74.0) and hyperbilirubinemia (coefficient of variation: 70.1). Even among newborns with two or more mild conditions, rate of NICU admission 


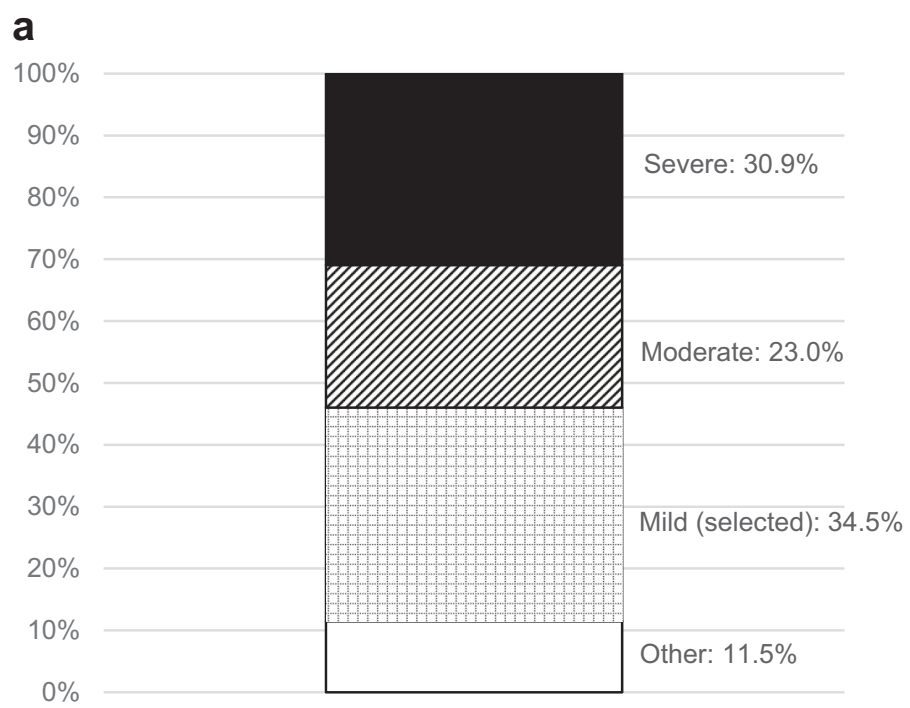

b

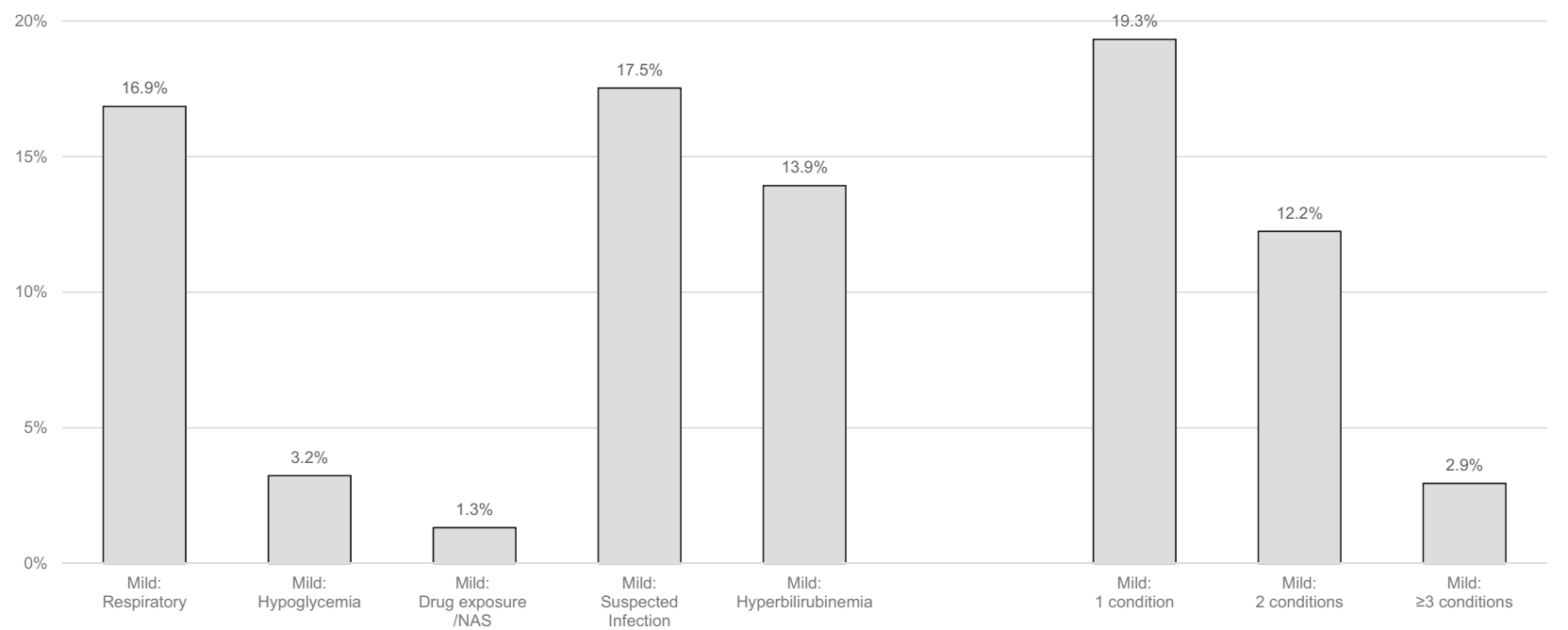

Fig. 2 Distribution of diagnosis severity and category among infants admitted to neonatal intensive care unit in the overall sample. a By severity of the conditions. "Other" includes infants with diagnoses not meeting the definition for severe, moderate or the five

varied considerably across hospitals (coefficient of variation: 51.6).

NICU admission rates among the mild conditions were generally positively correlated (Appendix B). For instance, among the hospitals, rates of NICU admission for mild respiratory conditions and suspected infection had a correlation coefficient of $0.45(p=0.007)$. Admission rates between all pairs of mild conditions except for two had significant positive correlations ranging from 0.43 to 0.64 , suggesting moderate to high correlations [22]. Thus, hospitals that had high admission rates for one mild condition selected mild conditions. b By different categories of mild conditions (categories not mutually exclusive) and number of mild conditions. NAS neonatal abstinence syndrome.

tended to have higher rates of admission for a majority of the other mild conditions.

For newborns with mild conditions, multivariable regression analysis identified several maternal, neonatal, and hospital characteristics that were significantly associated with the likelihood of NICU admissions (Table 3). More advanced maternal age, nulliparity, as well as elevated maternal weight above a normal body mass index were associated with a higher likelihood of NICU admission. Infants with Medicaid coverage were more likely to be admitted to NICUs than those with private insurance 
Table 2 Proportion of births with a given condition that were admitted to a neonatal intensive care unit, overall sample, and variation across hospitals.

\begin{tabular}{|c|c|c|c|c|c|c|c|}
\hline \multirow{2}{*}{$\begin{array}{l}\text { Diagnosis severity and } \\
\text { category }\end{array}$} & \multicolumn{4}{|c|}{ Overall sample } & \multicolumn{3}{|c|}{ Variation across hospitals } \\
\hline & $\begin{array}{l}\text { \# of births } \\
\text { with a given } \\
\text { condition }\end{array}$ & $\begin{array}{l}\text { Length of } \\
\text { hospital stay, } \\
\text { mean } \pm \text { SD }\end{array}$ & $\begin{array}{l}\text { \# of births with a } \\
\text { given condition that } \\
\text { were admitted } \\
\text { to NICU }\end{array}$ & $\begin{array}{l}\% \text { Admitted } \\
\text { to NICU }\end{array}$ & $\begin{array}{l}\# \text { of } \\
\text { hospitals }^{\mathrm{a}}\end{array}$ & Median (IQR) & $\begin{array}{l}\text { Coefficient of } \\
\text { variation }\end{array}$ \\
\hline Severe & 6167 & $8.8 \pm 13.8$ & 5423 & 87.9 & 35 & $87.8(81.5-91.5)$ & 8.8 \\
\hline Moderate & 5579 & $7.6 \pm 6.2$ & 4040 & 72.4 & 34 & $73.5(65.0-79.8)$ & 14.1 \\
\hline \multicolumn{8}{|l|}{ Mild } \\
\hline Respiratory & 7460 & $2.4 \pm 0.9$ & 2954 & 39.6 & 35 & $37.5(20.9-50.9)$ & 52.0 \\
\hline Hypoglycemia & 1734 & $3.0 \pm 2.6$ & 567 & 32.7 & 26 & $31.4(25.0-43.2)$ & 46.4 \\
\hline $\begin{array}{l}\text { Drug exposure/ } \\
\text { NAS }\end{array}$ & 1181 & $2.8 \pm 2.9$ & 231 & 19.6 & 12 & $15.9(9.2-23.8)$ & 52.4 \\
\hline Suspected infection & 20,222 & $2.3 \pm 1.4$ & 3072 & 15.2 & 35 & $17.4(8.4-32.8)$ & 74.0 \\
\hline Hyperbilirubinemia & 53,630 & $2.6 \pm 1.1$ & 2441 & 4.6 & 35 & $3.9(2.4-8.3)$ & 70.1 \\
\hline 1 mild condition & 64,941 & $2.5 \pm 1.2$ & 3387 & 5.2 & 35 & $4.7(2.3-7.40)$ & 82.9 \\
\hline$\geq 2$ mild conditions & 9169 & $3.2 \pm 2.5$ & 2663 & 29.0 & 34 & $36.4(19.8-46.4)$ & 51.6 \\
\hline
\end{tabular}

$I Q R$ interquartile range, $N A S$ neonatal abstinence syndrome, $S D$ standard deviation.

${ }^{a}$ Limited to hospitals with at least 25 births with the given condition.

(adjusted $\mathrm{OR}=1.20,95 \% \mathrm{CI}: 1.10-1.32)$. Infants with early (35-37 weeks) or late (41 weeks) gestational ages were more likely to be admitted than those of 40 weeks gestation (adjusted OR ranging from 1.14 to 5.41). Similarly, newborns with birth weight below $2500 \mathrm{~g}$ (adjusted $\mathrm{OR}=2.85$, 95\% CI: 2.50-3.23) or greater than or equal to $4000 \mathrm{~g}$ (adjusted OR $=1.30,95 \%$ CI: $1.17-1.44$ ) were both more likely to be admitted than those with birthweights between 2500 and $3999 \mathrm{~g}$. Compared to newborns with hyperbilirubinemia, those with other mild conditions or with multiple mild conditions had an increased odds of being admitted with adjusted OR ranging from 3.31 for drug exposure/NAS to 76.24 for having three or more mild conditions. In addition, newborns delivered at hospitals with more NICU beds had a higher odds of admission (adjusted $\mathrm{OR}=1.97,95 \% \mathrm{CI}$ : 1.06-3.65, for newborns at hospitals with higher versus lower than the median number of NICU beds per 1000 live inborn births). Other institutional attributes (level of NICU, type of hospital ownership, teaching status, and multihospital system affiliation) were not significantly associated with the likelihood of NICU admission for newborns with mild conditions.

\section{Discussion}

Our evaluation of data from linked birth certificate and newborn hospital discharge records demonstrated high variability in overall NICU admission rates, as well as NICU admission rates for mild conditions, across California hospitals. One third of all NICU admissions had a mild diagnosis condition. Furthermore, births with multiple mild diagnoses or at hospitals with more NICU beds had a higher likelihood of NICU admission.

The need for NICU admission of infants with severe or moderate diagnoses is likely more obvious to clinicians; however, the decision to admit infants in mild diagnosis categories may be more open to individual clinician judgment. The trend toward increasing NICU admission rates over the last several years without obvious changes in newborn disease severity [1] along with our finding that a substantial portion of NICU admissions are in the discretionary admission zone with high interhospital variability, suggest potential overuse of NICUs. This is consistent with a previous study of 19 hospitals showing that $0-59.4 \%$ of NICU admissions for infants of 35-42 weeks' gestation and $\geq 2500$ g were without an absolute or relative identified cause [23]. There is a need to better understand admission criteria and their influencing factors for mild diagnoses in current practice. Such information will allow for more informed strategies to reduce unnecessary NICU admissions.

In order to better understand reasons for variability, it may be beneficial for future studies to examine and compare practices at hospitals with high versus low rates of NICU admission for the mild diagnoses in more detail. Hospitals may differ in factors such as nursing comfort and training with newborn care, presence of a transitional nursery, physician staffing hours, admission protocols, and other systems factors that could drive the variation in admission rates that we observed. Financial incentives may also play a role. We found that babies born at hospitals with more NICU beds were more likely to have NICU admissions, which is 
Table 3 Adjusted association of patient and hospital characteristics with the likelihood of neonatal intensive care unit admission, among newborns with mild conditions.

\begin{tabular}{ll}
\hline Characteristics & $\begin{array}{l}\text { Odds ratio } \\
(95 \% \text { confidence interval) }\end{array}$ \\
\hline
\end{tabular}

Maternal age (years)

$\begin{array}{ll}<18 & 0.88(0.70,1.10) \\ 18-24 & 0.88(0.80,0.97) \\ 25-29 & \text { Reference } \\ 30-34 & 1.00(0.91,1.09) \\ 35-39 & 1.18(1.07,1.30) \\ \geq 40 & 1.20(1.03,1.40)\end{array}$

Race/ethnicity

Non-Hispanic white

Reference

Non-Hispanic black

$1.07(0.93,1.24)$

Hispanic

$0.95(0.88,1.04)$

Other

Unknown

$0.93(0.85,1.02)$

$0.08(0.01,0.70)$

Primary payer

Private insurance

Medicaid

Reference

Self-pay

$1.20(1.10,1.32)$

$1.03(0.78,1.37)$

$1.69(1.38,2.07)$

Gestational age (weeks)

35
36
37
38
39
40
41
42

$5.41(4.52,6.49)$

$2.27(1.96,2.63)$

$1.38(1.23,1.56)$

$1.05(0.95,1.17)$

$0.98(0.89,1.06)$

Reference

$1.14(1.01,1.29)$

$1.05(0.73,1.53)$

Maternal body mass index

$\begin{array}{ll}\text { Underweight } & 0.90(0.75,1.08) \\ \text { Normal } & \text { Reference } \\ \text { Overweight } & 1.11(1.02,1.20) \\ \text { Obese } & 1.10(0.999,1.20) \\ \text { Morbidly obese } & 1.36(1.15,1.62) \\ \text { Unknown } & 1.14(0.98,1.33)\end{array}$

Birth weight (grams)

$\begin{array}{ll}1500-2499 & 2.85(2.50,3.23) \\ 2500-3999 & \text { Reference } \\ \geq 4000 & 1.30(1.17,1.44)\end{array}$

Parity

$\begin{array}{ll}0 & \text { Reference } \\ 1 & 0.66(0.61,0.72) \\ \geq 2 & 0.64(0.58,0.70) \\ \text { Unknown } & 1.01(0.19,5.50)\end{array}$

Unknown $1.01(0.19,5.50)$
Table 3 (continued)

\begin{tabular}{ll}
\hline Characteristics & $\begin{array}{l}\text { Odds ratio } \\
\text { (95\% confidence interval) }\end{array}$ \\
\hline
\end{tabular}

Apgar score (5 min)

$\begin{array}{ll}0-6 & 8.06(6.11,10.62) \\ 7-10 & \text { Reference } \\ \text { Unknown } & 1.55(1.002,2.39) \\ \text { nenital malformations } & \\ \text { Yes } & 2.32(2.04,2.64) \\ \text { No } & \text { Reference }\end{array}$

Categories of mild conditions ${ }^{\mathrm{a}}$

$\begin{array}{ll}\text { Respiratory } & 28.01(25.17,31.18) \\ \text { Hypoglycemia } & 9.00(7.21,11.23) \\ \text { Drug exposure/NAS } & 3.31(2.40,4.56) \\ \text { Suspected infection } & 5.35(4.82,5.94)\end{array}$

Hyperbilirubinemia Reference

Two mild conditions $20.73(18.87,22.77)$

$\geq$ Three mild conditions $\quad 76.24(64.66,89.89)$

Teaching status

$\begin{array}{ll}\text { Teaching } & 1.29(0.64,2.59) \\ \text { Non teaching } & \text { Reference }\end{array}$

Type of ownership

Government (non federal) $1.14(0.45,2.89)$

Private non profit Reference

Private for-profit $\quad 0.98(0.31,3.11)$

Multihospital system affiliation
Yes
$1.82(0.80,4.14)$
No
Reference

Level of NICU
III
Reference
IV
$0.69(0.26,1.83)$

Number of NICU beds per 1000 inborn live births
$<$ Median $^{\mathrm{b}}$
Reference
$\geq$ Median $^{\text {b }}$
$1.97(1.06,3.65)$

NAS neonatal abstinence syndrome, NICU neonatal intensive care unit. ${ }^{\mathrm{a}}$ For the purpose of this regression analysis, newborns were categorized into mutually exclusive groups indicating whether they had mild respiratory condition alone, hypoglycemia alone, drug exposure/NAS alone, suspected infection alone, hyperbilirubinemia alone, two of these mild conditions, or $\geq 3$ of these mild conditions. Newborns with hyperbilirubinemia alone were used as a reference group since that was the largest group.

${ }^{\mathrm{b}}$ Sample median number of NICU beds per 1000 inborn live births was 7.114 .

consistent with prior studies [24, 25]. Examination of different hospital processes may allow for creation of standardized protocols or system processes to help reduce unnecessary NICU admissions, and thereby decrease adverse financial, psychosocial, and downstream health effects. 
We also found that infants with more than one mild diagnosis category tended to have a higher odds of NICU admission. NICU admission for infants with one mild diagnosis may be considered more discretionary than for infants with two or more mild diagnoses. It is possible that clinician consideration of the contribution of several mild factors favors a setting of closer observation. However, it is not known that infants with these mild diagnosis categories, whether one or several, are better cared for in the NICU versus another setting such as a well-baby or transitional nursery. Although there is a spectrum of severity even amongst this mild category, the high variation seen across NICUs would imply that some NICUs are more likely to admit infants with milder disease severity. Thus, further work looking into outcomes of these infants admitted to the NICU versus monitored in the well-baby or transitional nurseries could be beneficial in understanding the best location of care.

We recognize several limitations of this study. First, NICU admissions measured on birth certificates may lack sufficient accuracy. Although we limited our analysis to the 35 hospitals that were previously shown to provide good quality data with regards to NICU admission rate [14] and supplemented the NICU admission indicator on birth certificate with other information suggestive of typical procedures performed in NICUs, we might still underestimate NICU admissions. However, our data showed good validity in several aspects: longer length of stay and higher hospital charges for infants that were admitted to NICU versus those that were not, and lower variation in NICU admission rates for severe and moderate conditions than milder conditions. Given that our focus was hospital variation, rather than the exact rate of NICU admissions, our findings should provide reasonable information to inform the discussion about interhospital differences in NICU admissions. Second, this study was also limited in that it relied on administrative data. Diagnosis codes were used for categorizing disease severity and categories which are less accurate than medical record data. Interhospital variation in ICD coding practices may also confound our findings. However, use of administrative data provides an opportunity to study variation across a large number of hospitals which is less feasible with manual medical record extraction. Third, we lacked information on potential variation in hospital capacity, i.e., the ability to house patients in a transition nursery or on a pediatric floor, which could confound the variability in NICU admission rates we observed. Fourth, our analysis was based on data in 2010-2012. Changes in practice style are occurring since the time of this data (e.g., more widespread adoption of the Kaiser sepsis calculator [26], creation of the Northern California Neonatal Consortium Jaundice guideline [27], and increased evidence supporting housing of NAS babies in well-baby nurseries with their mothers
[28]). These efforts would likely lead to decreased NICU admission rates in more recent years and call for additional research using more updated data. Lastly, although our study focused on hospitals with level III-IV NICUs, some of these hospitals might also have level II NICU beds. Since the birth certificate reporting guidelines exclude level II NICU admissions, some of our observed variability in birth certificate documented NICU admission rates could arise from differential designation of NICU beds/patients as level II versus III-IV across hospitals. Future research using a data set including level II admission information would help evaluate this potential confounding factor.

Overall, among the infants admitted to the 35 California NICUs in 2010-2012 between 35 and 42 weeks gestation with a birth weight over $1500 \mathrm{~g}$, one third had mild diagnoses. There was high interhospital variation in overall NICU admission rates, as well as NICU admission rates for mild diagnoses. Furthermore, having more NICU beds was associated with a higher likelihood of NICU admission. These findings suggest potential overuse of NICUs by some hospitals for mild conditions. Further investigation is warranted to better understand the potential reasons for this variation in order to inform strategies to reduce unnecessary NICU admissions.

Acknowledgements Results from this study were accepted for poster presentation at the Pediatric Academic Societies meeting, May 2-5, 2020, in Philadelphia, PA which was canceled due to COVID-19. Results from this study have been submitted to the American Academy of Pediatrics national conference for consideration of presentation.

Funding This project was supported by grant number R01HS023801 from the Agency for Healthcare Research and Quality. The content is solely the responsibility of the authors and does not necessarily represent the official views of the Agency for Healthcare Research and Quality. ESH was supported by the Stanford Maternal \& Child Health Research Institute.

Author contributions ESH conceptualized the study and drafted the initial paper; HCL conceptualized the study and helped draft the initial paper; XX conceptualized the study, conducted data analysis, and helped draft the initial paper; and all authors provided interpretation of data, reviewed, and revised the paper critically for important intellectual content, and approved the final paper as submitted.

\section{Compliance with ethical standards}

Conflict of interest The authors declare that they have no conflict of interest.

Publisher's note Springer Nature remains neutral with regard to jurisdictional claims in published maps and institutional affiliations.

\section{References}

1. Harrison W, Goodman D. Epidemiologic trends in neonatal intensive care, 2007-12. JAMA Pediatr. 2015;169:855-62. 
2. Schulman J, Braun D, Lee HC, Profit J, Duenas G, Bennett MV, et al. Association between neonatal intensive care unit admission rates and illness acuity. JAMA Pediatr. 2018;172:17-23.

3. Edwards EM, Horbar JD. Variation in use by NICU types in the United States. Pediatrics. 2018;142:e20180457.

4. Harrison WN, Wasserman JR, Goodman DC. Regional variation in neonatal intensive care admissions and the relationship to bed supply. J Pediatr. 2018;192:73-9.e4.

5. Goodman DC, Little GA, Harrison WN, Moen A, Mowitz ME, Ganduglia Cazaban C, et al. (eds.) The Dartmouth Atlas of Neonatal Intensive Care. Lebanon, NH: The Dartmouth Institute of Health Policy \& Clinical Practice, Geisel School of Medicine at Dartmouth; 2019.

6. Phibbs CS, Schmitt SK, Cooper M, Gould JB, Lee HC, Profit J, et al. Birth hospitalization costs and days of care for mothers and neonates in California, 2009-11. J Pediatr. 2019;204:118-25.e14.

7. Sekar KC. Iatrogenic complications in the neonatal intensive care unit. J Perinatol. 2010;30:S51-6.

8. Polin RA, Denson S, Brady MT. Committee on fetus and newborn, committee on infectious diseases. Epidemiology and diagnosis of health care-associated infections in the NICU. Pediatrics. 2012;129:e1104-9.

9. Hannan KE, Juhl AL, Hwang SS. Impact of NICU admission on Colorado-born late preterm infants: breastfeeding initiation, continuation and in-hospital breastfeeding practices. J Perinatol. 2018;38:557-66.

10. Wigert H, Johansson R, Berg M, Hellström AL. Mothers' experiences of having their newborn child in a neonatal intensive care unit. Scand J Caring Sci. 2006;20:35-41.

11. Hynan MT, Mounts KO, Vanderbilt DL. Screening parents of high-risk infants for emotional distress: rationale and recommendations. J Perinatol. 2013;33:748-53.

12. California Office of Statewide Health Planning and Development. Linked birth files. OSHPD. 2020. https://oshpd.ca.gov/data-andreports/research-data-request-information/.

13. Herrchen B, Gould JB, Nesbitt TS. Vital statistics linked birth/ infant death and hospital discharge record linkage for epidemiological studies. Comput Biomed Res. 1997;30:290-305.

14. Haidari ES, Lee HC, Illuzzi JL, Lin H, Xu X. Utility of birth certificate data for evaluating hospital variation in admissions to NICUs. Hosp Pediatr. 2020;10:190-4.
15. Centers for Disease Control and Prevention. National Center for Health Statistics. National VItal Statistics System. Guide to completing the facility worksheets for the certificate of live birth and report of fetal death. 2019;50. https://www.cdc.gov/nchs/data/dvs/ GuidetoCompleteFacilityWks.pdf.

16. Unexpected Complications in Term Newborns I California Maternal Quality Care Collaborative. 2019. https://www.cmqcc. org/focus-areas/quality-metrics/unexpected-complications-termnewborns.

17. National Quality Forum. Unexpected complications in term newborns. 2019. http://www.qualityforum.org/qps/0716.

18. The Joint Commission. PC-06 measure information form. 2019. https://manual.jointcommission.org/releases/TJC2019A/ MIF0393.html.

19. AHA Annual Survey Database ${ }^{\mathrm{TM}}$ I AHA Data. 2020. https://www. ahadata.com/aha-annual-survey-database.

20. California Perinatal Quality Care Collaborative. 2020. https://www. cpqcc.org/. Accessed July 16, 2020.

21. Centers of Medicare and Medicaid Services. Hospital compare 30day death and readmission measures data. 2019. https://www. medicare.gov/hospitalcompare/Data/30-day-measures.html.

22. Cohen J. Statistical power analysis for the behavioral sciences. Elsevier; 1977. https://linkinghub.elsevier.com/retrieve/pii/ C2013010517X.

23. Ziegler KA, Paul DA, Hoffman M, Locke R. Variation in NICU admission rates without identifiable cause. Hosp Pediatr. 2016; 6:255-60.

24. Freedman S. Capacity and utilization in health care: the effect of empty beds on neonatal intensive care admission. Am Econ J Econ Policy. 2016;8:154-85.

25. Carroll AE. The concern for supply-sensitive neonatal intensive care unit care: if you build them, they will come. JAMA Pediatr. 2015;169:812-3.

26. Dhudasia MB, Mukhopadhyay S, Puopolo KM. Implementation of the sepsis risk calculator at an academic birth hospital. Hosp Pediatr. 2018;8:243-50.

27. Northern CA Neonatology Consortium. NCNC Jaundice Guideline. 2020. https://www.phototherapyguidelines.com/.

28. Clemans-Cope L, Holla N, Lee HC, Cong AS, Castro R, Chyi L, et al. Neonatal abstinence syndrome management in California birth hospitals: results of a statewide survey. J Perinatol. 2020;40:463-72. 